\title{
Adjusting microbiome profiles for differences in microbial load by spike-in bacteria
}

\author{
Frank Stämmler ${ }^{1,2^{*}}$ (D), Joachim Gläsner ${ }^{2}$, Andreas Hiergeist ${ }^{2}$, Ernst Holler ${ }^{3}$, Daniela Weber ${ }^{3}$, Peter J. Oefner ${ }^{4}$,
} André Gessner ${ }^{2}$ and Rainer Spang ${ }^{1}$

\begin{abstract}
Background: Next-generation $16 \mathrm{~S}$ ribosomal RNA gene sequencing is widely used to determine the relative composition of the mammalian gut microbiomes. However, in the absence of a reference, this does not reveal alterations in absolute abundance of specific operational taxonomic units if microbial loads vary across specimens.

Results: Here we suggest the spiking of exogenous bacteria into crude specimens to quantify ratios of absolute bacterial abundances. We use the $16 \mathrm{~S}$ rDNA read counts of the spike-in bacteria to adjust the read counts of endogenous bacteria for changes in total microbial loads. Using a series of dilutions of pooled faecal samples from mice containing defined amounts of the spike-in bacteria Salinibacter ruber, Rhizobium radiobacter and Alicyclobacillus acidiphilus, we demonstrate that spike-in-based calibration to microbial loads allows accurate estimation of ratios of absolute endogenous bacteria abundances. Applied to stool specimens of patients undergoing allogeneic stem cell transplantation, we were able to determine changes in both relative and absolute abundances of various phyla, especially the genus Enterococcus, in response to antibiotic treatment and radio-chemotherapeutic conditioning.
\end{abstract} Conclusion: Exogenous spike-in bacteria in gut microbiome studies enable estimation of ratios of absolute OTU abundances, providing novel insights into the structure and the dynamics of intestinal microbiomes.

Keywords: Microbial load, Spike-in bacteria, $16 \mathrm{~S}$ rRNA gene sequencing, Standardization, Microbiome profiling, Bacterial communities, Community analysis

\section{Background}

The human intestinal tract is populated by an ecological community of microorganisms, the gut microbiome. This complex community plays an important role in health and disease [1-7] and varies greatly among individuals. Next generation sequencing of the $16 \mathrm{~S}$ rRNA gene allows profiling of the bacterial and archaeal components of the gut microbiome at unprecedented precision and depth [8-10]. Computational tools such as QIIME [11] and mothur [12] cluster reads into operational taxonomic units (OTUs) [13], which may then be jointed into taxonomic groups at the genus, family, order, class, and phylum level.

\footnotetext{
* Correspondence: Frank.Staemmler@klinik.uni-regensburg.de

${ }^{1}$ Chair of Statistical Bioinformatics, University of Regensburg, Am BioPark 9, 93053 Regensburg, Germany

${ }^{2}$ Institute of Clinical Microbiology and Hygiene, University Medical Centre,

Franz-Josef-Strauß-Allee 11, 93053 Regensburg, Germany

Full list of author information is available at the end of the article
}

Current studies focus on the relative abundance or proportions of OTUs $[14,15]$. As an example, a specific OTU may contribute $5 \%$ to microbiome A and $10 \%$ to microbiome $\mathrm{B}$ corresponding to a ratio of 1:2. If we further assume that the total number of bacteria or microbial load of in $\mathrm{A}$ is four times larger than in $\mathrm{B}$, the $5 \%$ in $\mathrm{A}$ account for twice as many bacteria as the $10 \%$ in $\mathrm{B}$, thus bringing the actual ratio to 2:1.

Antibiotic treatment, diet, and/or disease affect both microbial loads and compositions. For example, Holler et al. [16] observed that the relative abundance of the genus Enterococcus in stool specimens collected from patients undergoing allogeneic stem cell transplantation (ASCT) can increase from undetectable levels prior to ASCT to up to $94 \%$ after ASCT. More interestingly, this relative shift to Enterococcus was associated with an increased risk of acute gastrointestinal graft-versus-host 
disease (GI-GvHD). Without knowledge of total microbial load, however, it is impossible to infer whether this shift was the result of either an absolute increase in the number of Enterococcus or a decrease in the number of bacteria other than Enterococcus.

Application of synthetic spike-in standards allows for changing the profiles' reference points. The reference point of relative abundances is a fixed aliquot of $16 \mathrm{~S}$ rDNA. These profiles are insensitive to the microbial load of a stool specimen. Adding controlled amounts of spike-in material allows for rescaling the profiles such that the measured concentrations of the standard are constant across samples, making the spike-in standard the new reference point of the profiles and the profiles sensitive to microbial loads. Spike-in strategies featuring different GC contents and covering a wide concentration range in combination with appropriate normalization strategies have already been proposed to correct for library preparation and nuisance technical effects in the inference of gene expression levels from RNA-Seq experiments [17]. This approach, as well as similar schemes employed in proteomics [18] and metabolomics, [19] adds the spike-in standards to transcriptomes, proteomes and metabolomes only after cell lysis and extraction of mRNA, proteins and metabolites, respectively, and thus do not allow correction of variation originating from these critical experimental steps. Recently Jones et al. [20] suggested using whole cell spike-in controls for monitoring this technical variability in the field of microbiome research.

Extending their results, we here suggest the addition of exogenous viable spike-in bacteria to rescale the read counts of endogenous bacteria. We call this protocol spikein-based calibration to total microbial load (SCML), and test it in a dilution experiment with defined absolute spikein bacteria abundances against serially diluted background microbiomes. Moreover, we reconsider the emergence of Enterococcus as the predominant genus in ASCT using SCML.

\section{Results}

\section{Choice of spike - in bacteria}

We used Salinibacter ruber (S. ruber, GenBank ID: CP000159), an extreme halophilic bacterium found in hypersaline environments [21], Rhizobium radiobacter ( $R$. radiobacter, GenBank ID: ASXY01000000), a nonphytopathogenic member of the Biovar I group of Agrobacterium found in the soil and the plant rhizosphere [22], as well as the thermo-acidophilic, endospore forming soil bacterium Alicyclobacillus acidiphilus (A. acidiphilus, GenBank ID: PRJDB697) [23]. These eubacteria belong to different phyla typically found in mammalian faecal microbiomes. They do not exist in the gut microbiome under physiological conditions and are well distinguishable from the bacteria in the gut using $16 \mathrm{~S}$ rRNA gene sequencing. Whole bacteria are spiked-in at fixed amounts. 16S rRNA gene copy numbers per genome vary between these species $(1,4$ and 6 rRNA gene copies per genome for $S$. ruber, $R$. radiobacter and $A$. acidiphilus, respectively). We quantify spike-in concentrations and measured concentrations relative to $16 \mathrm{~S}$ rRNA gene copies. Hence, if we say that we spiked-in bacteria in the same amounts, this means that the number of $16 \mathrm{~S}$ rRNA copies, but not necessarily the number of bacterial cells is identical.

\section{Design of spike-in calibration experiments}

Experiments were performed with spike-in bacteria whose absolute abundance was defined by design in increasingly diluted gut microbiomes. The dilution simulates nonconstant microbial loads.

S. ruber, $R$. radiobacter and A. acidiphilus were spiked into each of 36 aliquots of pooled murine stool samples. While $A$. acidiphilus and $R$. radiobacter were spiked into these samples at variable amounts, that of $S$. ruber was kept constant. S. ruber was used to measure microbial loads, while $A$. acidiphilus and $R$. radiobacter were used to validate the SCML approach. The precision of the spike-ins was independently validated using quantitative real time PCR (qRT-PCR). Importantly, this analysis also verified that all three bacteria were in fact not present in the pooled murine stool (Additional file 1: Table S1). Additional file 2: Table S2 summarizes the design of the validation experiment.

To validate the spike-in assay we compare calibrated ratios of observed reads with the expected ratios defined by the experimental design. The experimental design controls microbial loads at several levels:

(i) For each sample, we have expected total microbial loads defined by the stool dilution factor and the spike-in concentrations. (ii) For each of the two spike-ins A. acidiphilus and $R$. radiobacter we have expected within-species ratios of concentrations for every pair of samples (intraOTU comparison). (iii) For every pair of samples we have expected inter-species ratios between the two spike-ins both within and across samples (inter-OTU comparison). (iv) For all taxonomic units of the background microbiome we have expected abundance ratios defined by the dilution factor and the spike-in concentrations.

\section{The three spike-in bacteria yield different read turnouts but correlate well with microbial loads}

Figure 1a shows linear relationships between the spikedin $16 \mathrm{~S}$ rDNA copies (x-axis in $\log _{2}$ scale) of $A$. acidiphilus and $R$. radiobacter, respectively, and the resulting $\log _{2}$ read counts. The total number of spike-in reads increases with dilution of the background microbiome. Simultaneously, as a constant amount of $S$. ruber was 


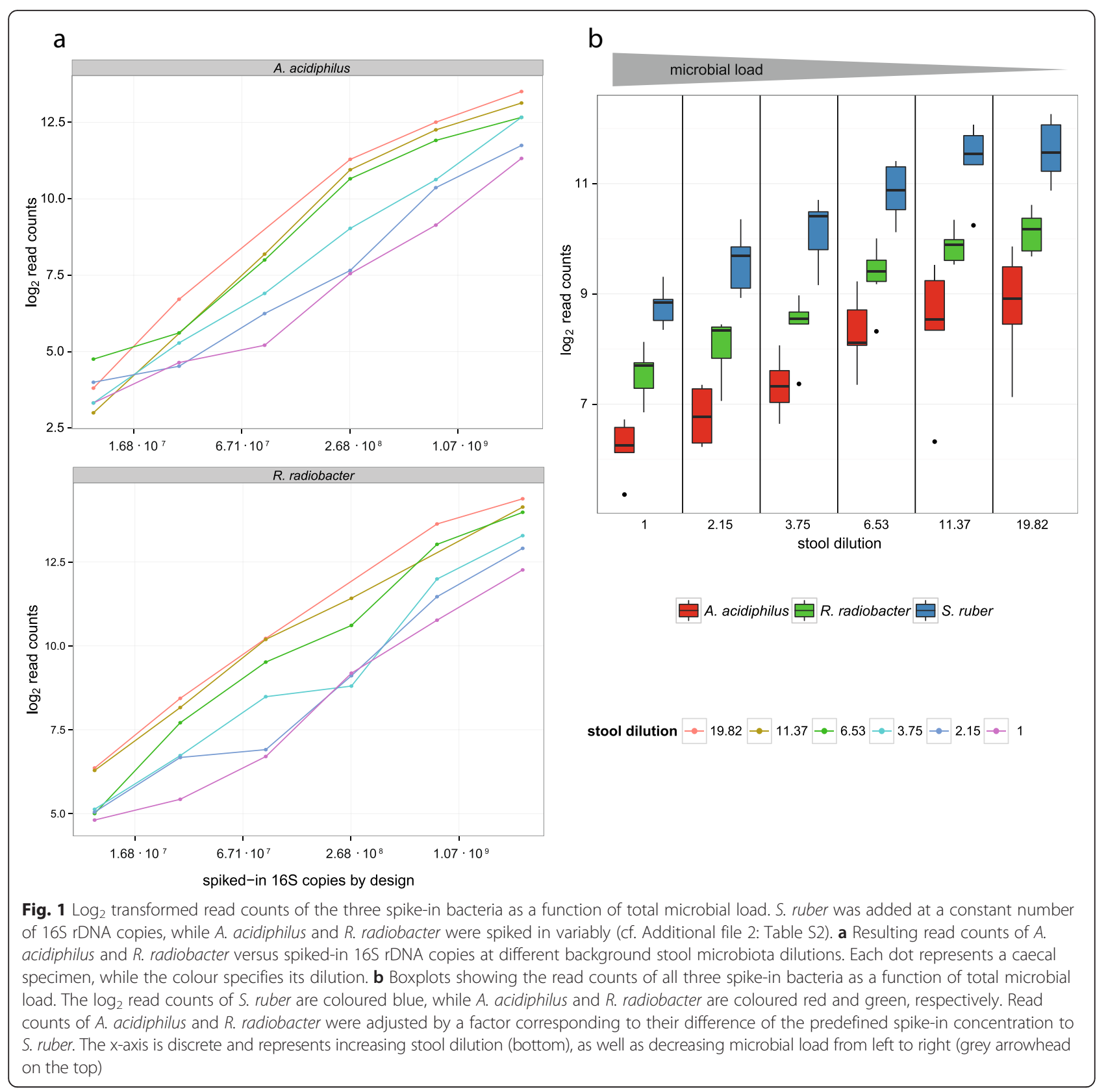

added to each sample, the portion of the spike-in bacteria increases (Fig. 1b). As a result, the read count assigned to a spike-in OTU is expected to inversely correlate with the total microbial load.

Figure 1b shows box plots of the $\log _{2}$ transformed read counts of S. ruber, R. radiobacter and A. acidiphilus as a function of microbial loads across all 36 samples. The counts were adjusted for their varying spike-in concentrations by design. For example, if in an experiment the concentration of the $A$. acidiphilus spike-in was only $50 \%$ of that of $S$. ruber, the A. acidiphilus counts were doubled. After adjustment of $A$. acidiphilus and $R$. radiobacter, we observe an inverse correlation of $\log _{2}$ spike-in counts with the microbial load (reciprocal dilution factor) for all three spike-in bacteria (Fig. 1b). In detail there is a correlation of $\mathrm{r}=-0.834$ for S. ruber, $\mathrm{r}=-0.795$ for $R$. radiobacter (adjusted) and $\mathrm{r}=-0.725$ for $A$. acidiphilus (adjusted). Additionally, we observe that the three bacteria have notably different read yields, with $S$. ruber showing the highest counts.

\section{SCML yields almost unbiased estimates of ratios of absolute abundances within taxonomic units}

For comparing SCML to standard relative abundance analysis, we generated two data sets by scaling the read counts with respect to two different reference points: 
First, we scaled the observed read counts relative to the library sizes. This gives us the standard relative abundances (standard data). In a second data set we scaled the same counts relative to the spike-in reads of $S$. ruber (SCML data).

We first compared the data for A. acidiphilus and $R$. radiobacter separately. By design the expected ratio for $A$. acidiphilus and $R$. radiobacter between every pair of samples is known. Figure 2 shows the observed intersample ratios for both data sets as a function of expected ratios. Plot (a) was created using standard data, while plot (b) was created using SCML data. We observe a reduced systematic error in (b) when comparing the data trend to the identity line (purple). The standard data shows systematically overestimated ratios in both directions. SCML reduced this bias. Moreover, we observe a high variability of estimated ratios, which was almost cut in half by SCML (Fig. 2c).

We next analysed the ratios for the background OTUs. By design, experimentally controlled ratios can be calculated from the dilution factor of the background microbiome. In contrast to $A$. acidiphilus and $R$. radiobacter the ratios derived from relative abundances (standard data) of these OTUs is zero by experimental design. Figure 3 shows the distribution of observed background ratios as a function of corresponding expected ratios. Plot (a) was created using standard data, while plot (b) was created using SCML data. As expected, ratios of relative abundances cannot capture shifts in microbial loads that do not affect the composition (Fig. 3a). In line with the previous observations, we observe a reduction of estimation variance when using SCML (Fig. 3c). Correlations between expected and observed $\log _{2}$ ratios were 0.359 and 0.833 for the standard data and the SCML data, respectively.

\section{SCML allows more accurate estimation of ratios than} calibrating for total 16S rRNA gene copies using qRT-PCR Quantification of the total number of 16S rRNA gene copies by qRT-PCR may be used to determine microbial loads. To compare the practicability of the latter with SCML we used a SYBR Green-based qPCR assay to quantify $16 \mathrm{~S}$ rDNA (Additional file 1: Table S1). Figure 4 shows observed and expected $\log _{2}$ ratios for
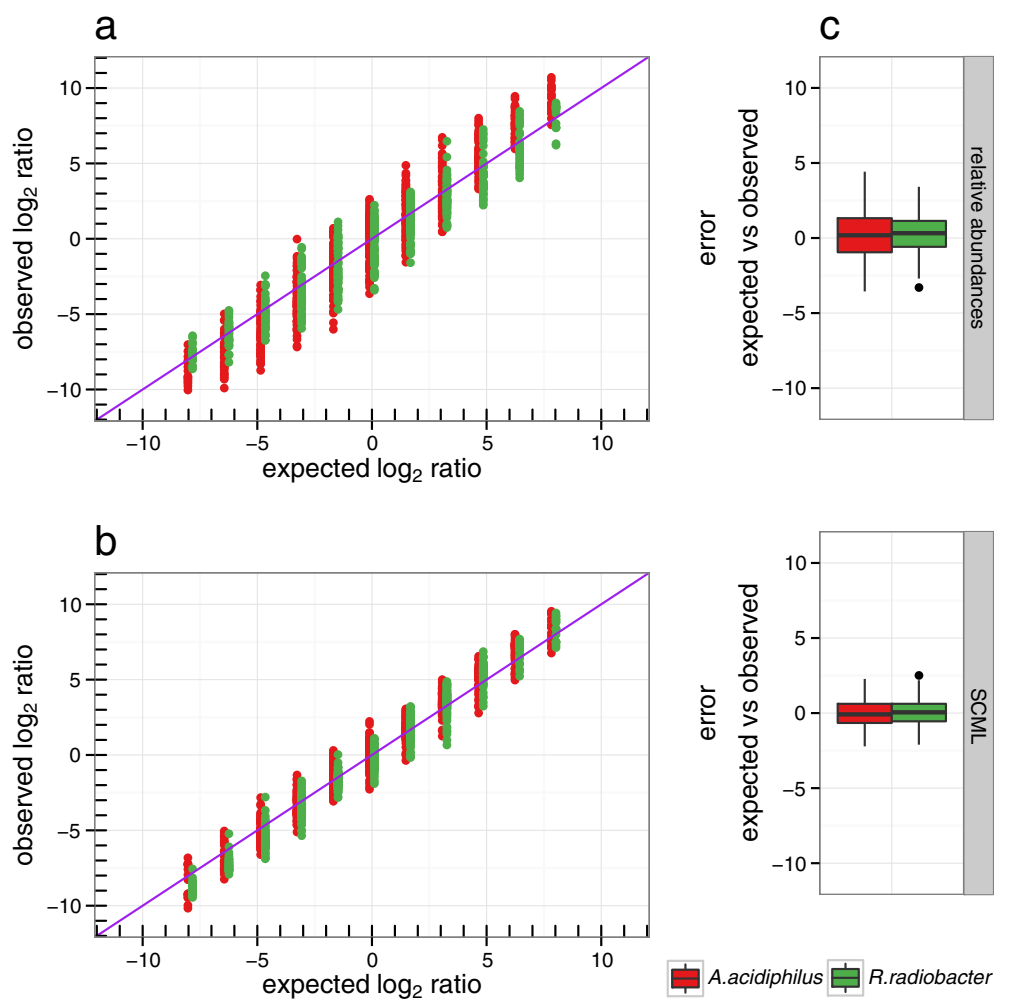

Fig. 2 Comparison of $\log _{2}$ ratios derived from relative abundances and after applying SCML to A. acidiphilus and R. radiobacter. Observed log 2 ratios versus expected $\log _{2}$ ratios of the spike-ins A. acidiphilus and $R$. radiobacter as derived from (a) relative abundances and (b) SCML by S. ruber for all pairwise sample comparisons. Both approaches were performed on the raw, not adjusted read counts of A. acidiphilus and $R$. radiobacter. The expected $\log _{2}$ ratios are calculated by the theoretical number of $16 \mathrm{~S}$ rDNA copies predetermined in the design of the validation experiment (cf. Additional file 2: Table S2). The purple diagonal represents the identity, which represents the expected $\log _{2}$ ratios by design. The box plots in (c) show the error between the expected and observed $\log _{2}$ ratios for both approaches. The smaller this error, the better calibrated the ratios are 


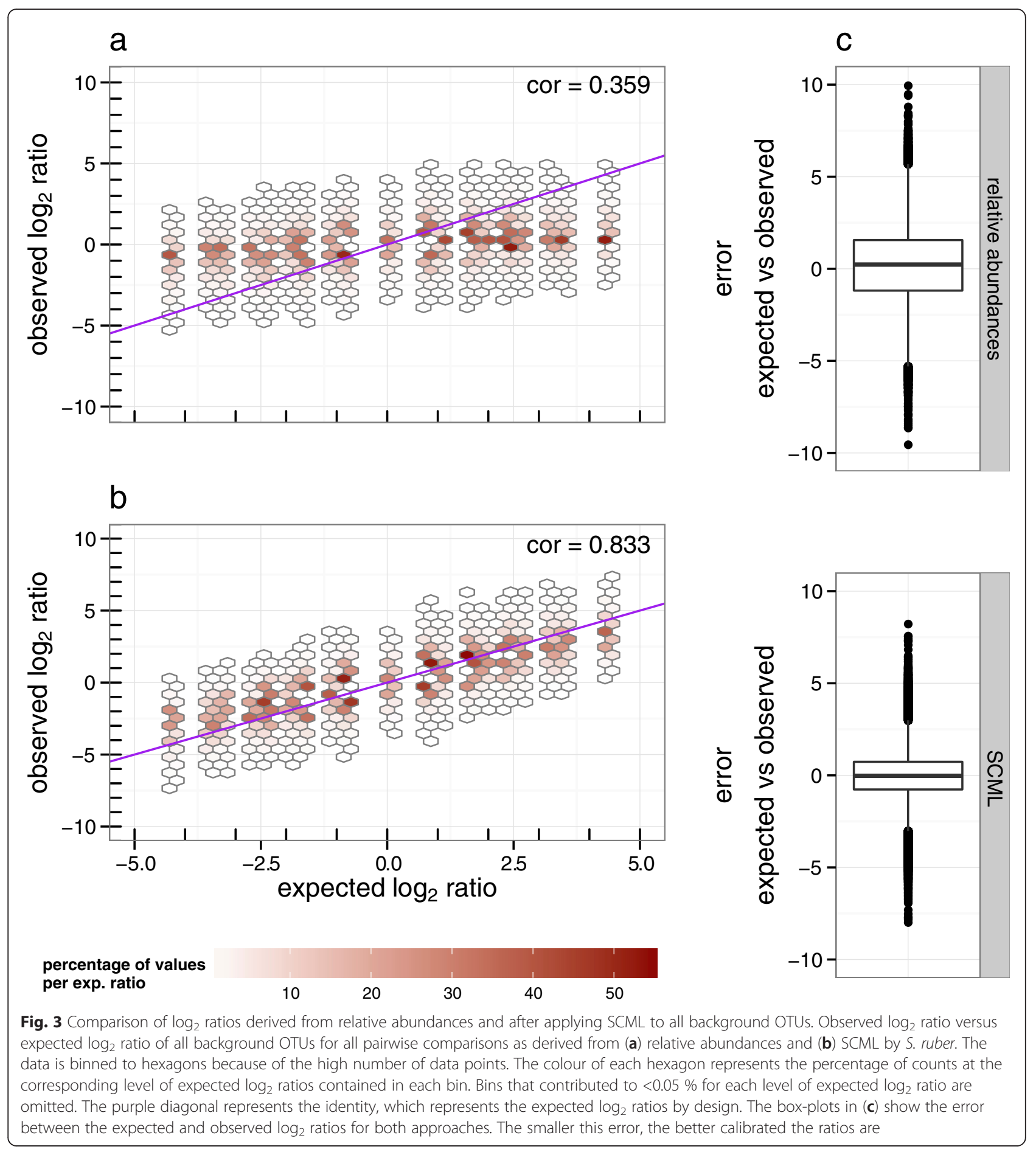

background OTUs using either (a) SCML or (b) rescaling to constant total 16S rRNA gene copies. It is apparent that observed ratios derived from SCML show higher concordance with the expected ratios regarding estimation bias and variance. Correlations between expected and observed $\log _{2}$ ratios were 0.717 and 0.833 for the qPCR and the SCML approach, respectively. These findings are also supported by an overall lower error between the observed and expected $\log _{2}$ ratios when derived from SCML (Fig. 4c). However, it has to be acknowledged that the SYBR Green-based quantification method of the bacterial load has not been explicitly compared to probe-based formats, so any limitations/imprecisions possibly resulting from the use of this universal detection format were not taken into account. 


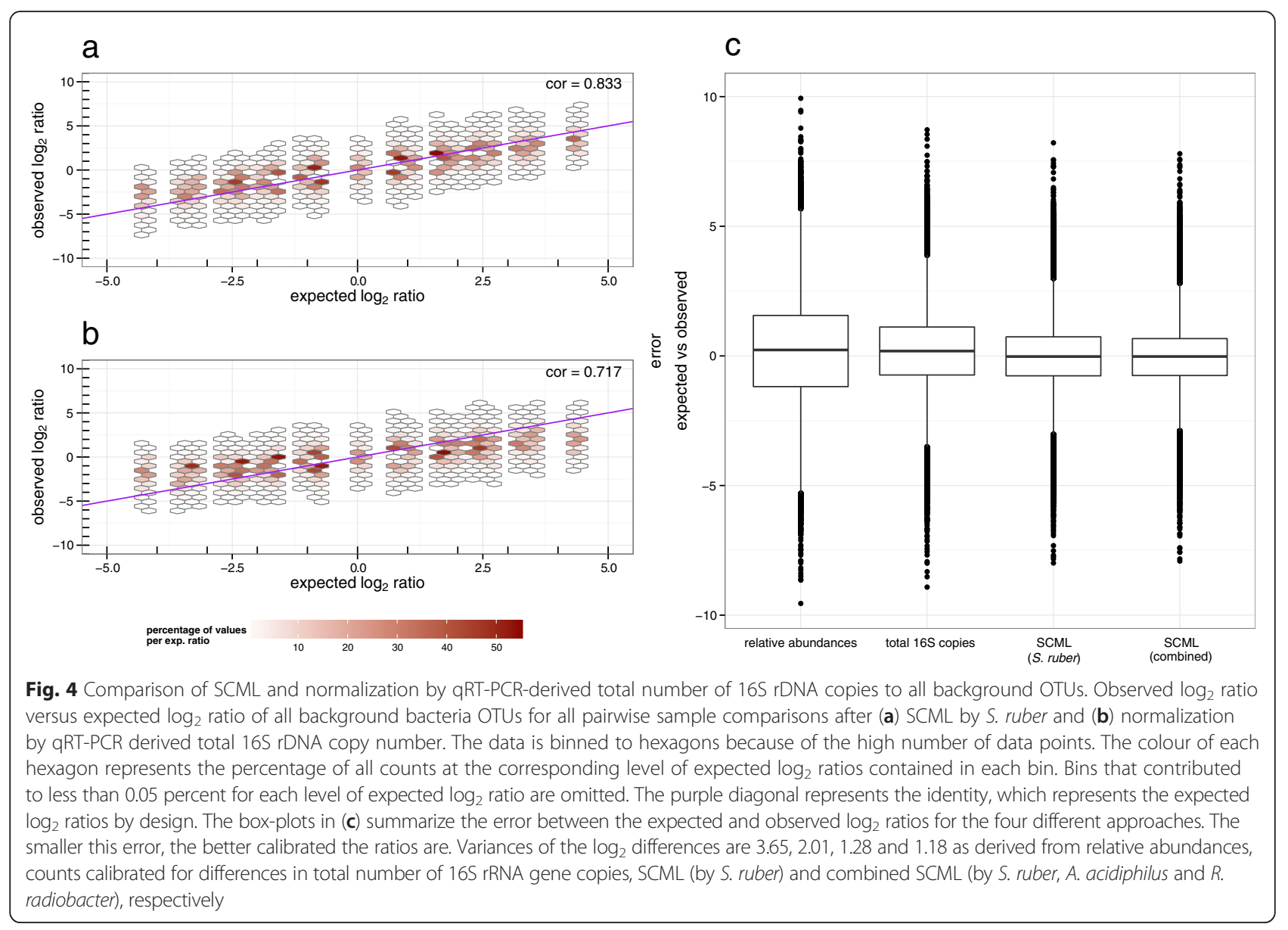

\section{Combining multiple spike-in bacteria reduces estimation errors}

Figure 1b shows that the adjusted counts of all three spike-in bacteria reciprocally correlated with microbial loads. We next investigated whether taking the sum of all three spike-in read counts further improves the estimates. Since $A$. acidiphilus and $R$. radiobacter were spiked in variable amounts we had to adjust their counts prior to using them for calibration. For example, if in an experiment the concentration of the $A$. acidiphilus spike-in was only $50 \%$ of that of $S$. ruber, the A. acidiphilus counts were doubled. We then used the sum of adjusted counts of all three spike-ins for calibration and repeated the analysis of the previous section. Figure $4 \mathrm{c}$ shows box-plots of the error between expected and observed $\log _{2}$ ratios for background OTUs based on relative abundances, read counts normalized by total $16 \mathrm{~S}$ rDNA copies, as well as based on the SCML data with $S$. ruber only and the combined counts of all three spikeins, respectively. The smaller this error, the better calibrated are the ratios of absolute abundances. Variances of these errors are 3.65, 2.01, 1.28 and 1.18, respectively. Thus, combined spike-ins yield a slightly increased precision compared to single spike-in usage. Correlations between expected and observed $\log _{2}$ ratios were 0.833 and 0.845 for the SCML and the combined SCML approach, respectively.

Calibration to microbial loads reveals absolute increase of Enterococcus in the intestine during allogeneic stem cell transplantation

Finally, we show that SCML expands our understanding of human microbiomes and their role in disease. Recently, a marked early loss of gastrointestinal microbiome diversity and an increase in relative abundance of members of the genus Enterococcus have been observed in the course of allogeneic stem cell transplantation (ASCT) and found to increase the risk of developing acute GI-GvHD [16, 24, 25]. Since the data had been generated without spike-in bacteria, it had not been possible to conclude whether the observed increase in relative abundance of Enterococcus was the result of an increase in absolute abundance of Enterococcus or of a decrease in abundance of other bacterial species.

Here we report on five patients, whose stool microbiomes were monitored prior to ASCT or on days 0 (d0), 7 (d7), and 14 (d14) after ASCT, respectively, using the proposed spike-in approach. Figure $5 \mathrm{a}$ shows the 


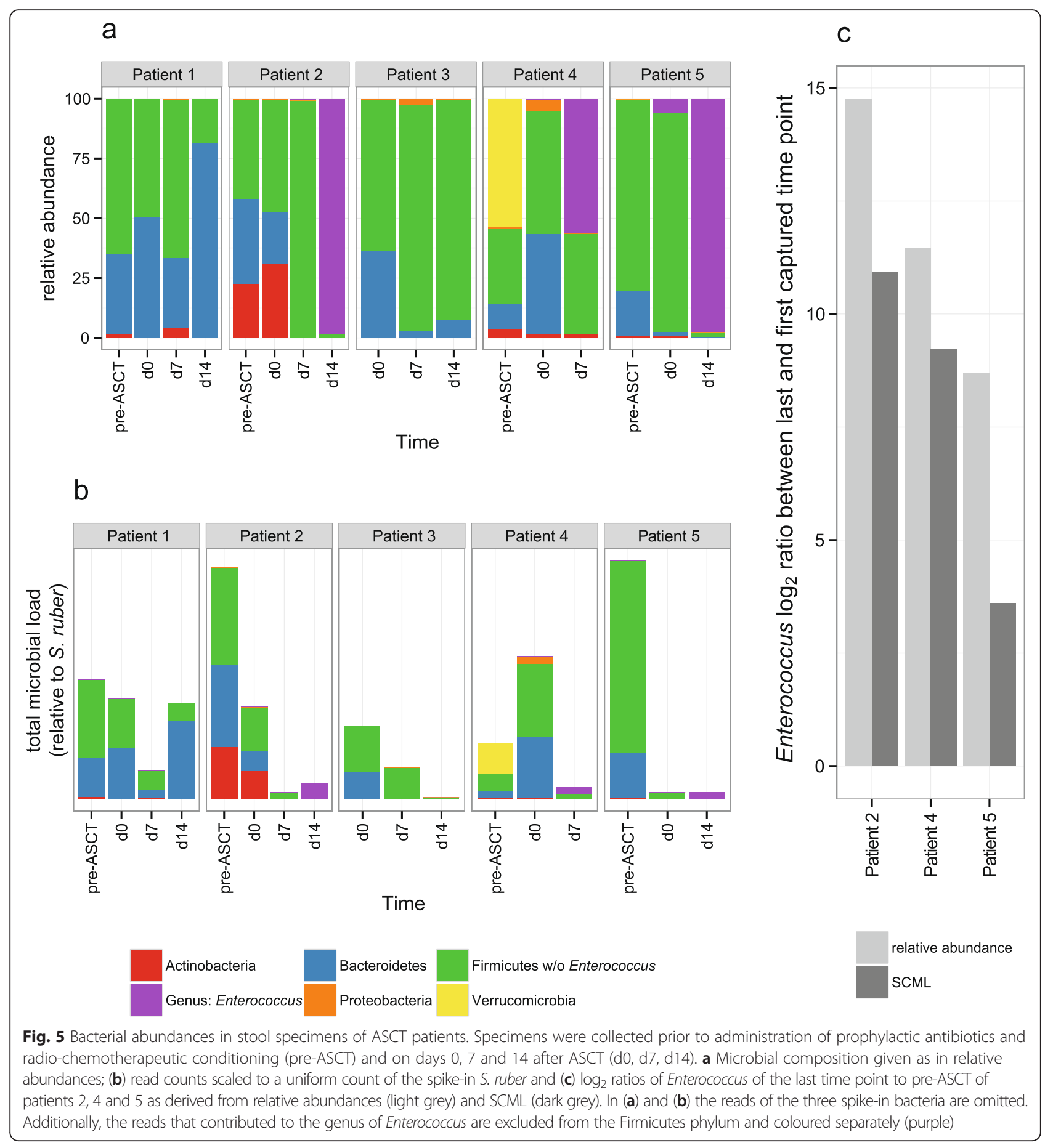

familiar diagram of relative microbiome composition without taking the spike-in bacteria into consideration. Reads contributing to the genus of Enterococcus are reported at genus resolution, while all other bacteria are shown on phyla resolution. In line with Holler et al. [16], we observe dramatic relative increases in Enterococcus abundance on days 7 and 14 after ASCT in three of the five patients. By scaling read counts to an even microbial load using the $S$. ruber counts, we observe marked changes in the microbial loads in the course of the treatment (Fig. 5b). Patient 5, for instance, shows an almost tenfold reduction of microbial load on day 14 after ASCT (S. ruber reads 4721) compared to preASCT (S. ruber reads 515). In our study, specimens dominated by Enterococcus generally have low microbial loads (Fig. 5b). We also observe an absolute increase in 
abundance of the genus Enterococcus in these microbiomes relative to the specimens collected before ASCT (Fig. 5c). Patients 2, 4 and 5 showed $\log _{2}$ ratios of Enterococcus between the last and first time point of 10.93, 9.22 and 3.60, respectively, employing SCML, compared to $\log _{2}$ ratios of $14.76,11.46$ and 8.69 based on standard data. This suggests that Enterococcus dominance is in fact associated with both a decrease in microbial load and a rise in absolute abundance of Enterococcus.

\section{Discussion}

Here we suggest the use of spike-in bacteria to calibrate multiple intestinal microbiome profiles to microbial loads (SCML). We employed $A$. acidiphilus, $R$. radiobacter and $S$. ruber as spike-in bacteria and demonstrated their excellent suitability for a comprehensive and informative profiling of gut microbiomes. Usually, these three bacteria are absent in the intestinal microbiomes of mammals, and their unique $16 \mathrm{~S}$ rRNA gene sequences cannot be mistaken for those of bacteria found in the gastrointestinal tract. All three bacteria are valid reporters of the actual microbial load. Thus SCML adds a new perspective to gut microbiome profiling that expands the common relative microbiome composition analysis.

Variability in microbial loads of intestines is a genuine and potentially clinically relevant biological feature that remains underutilised in standard protocols. On a more technical side, adding whole cells prior to lysis enables control for DNA recovery and pyrosequencing errors as a side benefit. Following this, the addition of exogenous spike-ins could also enhance other studies like wholegenome sequencing, qPCR-based quantification of pathogens as well as approaches using alternative marker genes [20, 26, 27].

Bacterial species compete for nutrients and can mutually displace each other, while others can only live in symbiosis. These dynamics of the intestinal ecosystem shape the structure of microbiome profiles [28, 29]. $\mathrm{Mu}-$ tually displacing, e.g. concurrent or antagonistic, species display anti-correlated profiles, while those of symbiotic species are correlated [30]. This theoretical consideration holds true for absolute numbers of bacteria. Interpreting the correlation structure on the basis of relative numbers can be misleading. If one species grows in absolute number, this will lead (i) to an increase of its fraction within the microbiome and (ii) to a decrease of the fractions of all other species. Hence, every change of a single species affects relative counts for all other species generating notorious anti-correlation between profiles of different species due to compositionality [31, 32]. Importantly, this effect is independent of ecological processes like displacement and symbiosis. Thus, profiles calibrated to ratios of total microbial loads provide a less disturbed assessment of the dynamics of the intestinal ecosystem.

We observed different sequence read yields for the three spike-in bacteria even upon addition of identical numbers of $16 \mathrm{~S}$ rDNA gene copies to mouse faeces sharing the same microbial load. Fortunately, this problem should only arise with comparisons of different species. As demonstrated, it does not affect the estimation of intra-species ratios between samples, where speciesspecific yields cancel.

There is a difference between absolute quantification and calibration of ratios of absolute abundances. The former needs calibration to a defined unit such as bacteria per volume. External spike-ins do not enable absolute quantification due to e.g. variable lysis efficiency across intestinal bacteria and variable $16 \mathrm{~S}$ rDNA copy numbers. In ratios the unit cancels. Hence, if in a comparison of the same OTU in two samples SCML calibrated values show a ratio of 2 , then the OTU is in fact represented (almost) twice as often. With standard relative data this is not the case, when microbial loads in these samples differ. Importantly, ratios between different OTUs are not calibrated by SCML. We can thus calibrate microbiome profiles to ratios of absolute abundance but not to absolute quantities of bacteria themselves.

A drawback of the spike-in approach is the propagation of PCR amplification errors from the spike-in bacterium to all other taxonomic units. Indeed, the spike-in counts can be affected by PCR amplification or sequencing errors. The earlier these errors occur, the more they could influence the final read tallies. By using these reads to calibrate microbial ratios, this error-derived variance propagates to all other taxonomic units. The calibration reduces bias, but inflates variance. One may attenuate this undesired effect by using multiple spike-in bacteria of fixed concentrations across samples and averaging or summing their counts as shown here.

\section{Conclusion}

In summary, we suggest that scaling of read counts to total microbial load and thereby calibrating the ratios of taxonomic units become standard routine in microbiome analysis, complementing the classical analysis of relative microbiome composition. SCML allows for accurate inter-sample comparison of microbiome profiles even in the presence of variation in microbial loads. Further, compared to the measurement of total number $16 \mathrm{~S}$ rDNA gene copies by qPCR, the spike-in bacteria are able to control additionally to DNA isolation and PCR amplification the sequencing process. Finally, our method allows a more robust identification of differentially abundant OTUs by enabling more accurate estimations of OTU-specific ratios. 


\section{Methods}

\section{Spike-in bacteria}

In this study we used Salinibacter ruber DSM $13855^{T}$, an extreme halophilic bacterium found in hypersaline environments [21], Rhizobium radiobacter DSM 30147 ${ }^{T}$, a non-phytopathogenic member of the Biovar I group of Agrobacterium found in soil and the plant rhizosphere [22], as well as the thermo-acidophilic, endospore forming soil bacterium Alicyclobacillus acidiphilus DSM $14558^{T}$ [23]. All bacteria were purchased from the DSMZ (German Collection of Microorganisms and Cell Cultures GmbH, Braunschweig, Germany). These eubacteria belong to different phyla typically found in mammalian faecal microbiomes, contributing to Bacteroidetes/Chlorobi group, Proteobacteria, and Firmicutes, respectively. They do not exist in the gut microbiome under physiological conditions and are well distinguishable from bacteria commonly found in the gut using $16 \mathrm{~S}$ rRNA gene sequencing. $S$. ruber and $R$. radiobacter are gram-negative bacteria, whereas $A$. acidiphilus is a spore-forming grampositive bacterium. The difference in the chemical constitution of the cell wall accounts for a specific susceptibility to the cell lysis protocol used. Spike-in bacteria were harvested in the late logarithmic/early stationary growth phase by centrifugation and subsequently resuspended in $5 \mathrm{ml}$ of sterile PBS buffer. Bacterial densities in suspensions were quantified by OD600 measurement using empirical conversion factors determined by direct microscopic cell counting. Accordingly, 1 OD600 unit corresponds to $4.6 \times 10^{9}$ cells $/ \mathrm{ml}$ for $S$. ruber, $1.4 \times 10^{9}$ cells/ $\mathrm{ml}$ for $R$. radiobacter, and $1.2 \times 10^{9}$ cells $/ \mathrm{ml}$ for $A$. acidiphilus, respectively. $16 \mathrm{~S}$ rRNA gene copy numbers per genome for the spike-in bacteria were obtained from the rrnDB database [33]. Six pools of bacterial mock communities containing $S$. ruber, $R$. radiobacter and A. acidiphilus were generated according to the scheme provided in Additional file 3: Table S3.

\section{Sample preparation and DNA extraction Mouse specimens}

For the validation experiment, cecum contents were collected from three 12-week-old male C57BL/6J mice (200 mg wet weight each), immediately suspended into $1 \mathrm{ml}$ of PBS, homogenized by means of the TissueLyser II (QIAGEN, Hilden, Germany), pooled, adjusted with PBS to a total volume of $4 \mathrm{ml}$, and split into seven aliquots of $550 \mu \mathrm{l}$ each. Six of these aliquots were diluted five times according to the scheme provided in Additional file 2: Table S2. Aliquot 7 was used as a non-spike control.

Sixty microliters of the corresponding spike-bacteria pool (whole cells) containing the desired number of $16 \mathrm{~S}$ rDNA copies (see Additional file 3: Table S3) were added to $250 \mu \mathrm{l}$ of all prepared, unlysed stool dilutions (see
Fig. 6, step 1) according to the scheme provided in Additional file 2: Table S2. Then, $180 \mu$ l of Bacterial Lysis Buffer (Roche, Mannheim, Germany) and $20 \mu \mathrm{l}$ Proteinase K (Fermentas GmbH, Sankt Leon-Rot, Germany) were added. Samples were incubated at $65{ }^{\circ} \mathrm{C}$ for $10 \mathrm{~min}$ followed by five cycles of freezing in liquid nitrogen $(1 \mathrm{~min})$ and boiling in hot water $\left(95^{\circ} \mathrm{C}, 1 \mathrm{~min}\right)$. Following the addition of $400 \mu \mathrm{l}$ of Bacterial Lysis Buffer and a mixture of $0.1-\mathrm{mm}$ and $2.5-\mathrm{mm}$ beads, samples were treated for $2 \mathrm{~min}$ at $30 \mathrm{~Hz}$ in the TissueLyser II. Subsequently, samples were heated at $95{ }^{\circ} \mathrm{C}$ for $15 \mathrm{~min}$ and centrifuged at $4{ }^{\circ} \mathrm{C}$ to pellet stool particles and beads (see Fig. 6, step 2). The final volume was adjusted to $1 \mathrm{ml}$ and DNA was extracted (see Fig. 6, step 3) by means of the MagNA Pure 96 instrument employing the MagNA Pure 96 DNA and Viral NA Large Volume Kit (Roche). Nucleic acids were quantified using the NanoDrop ND-1000 (Thermo Scientific, Wilmington, DE, USA).

\section{Human ASCT specimens}

With approval of the Ethics Committee of the University Medical Centre of Regensburg and after receipt of signed informed consent forms, stool specimens were collected at four different time points: prior to administration of prophylactic antibiotics and radio-chemotherapeutic conditioning, on days 0,7 , and 14, respectively, after ASCT. Stool specimens were stored at $-80{ }^{\circ} \mathrm{C}$ until analysis. Fifty mg (wet weight) of each stool specimen were suspended into $250 \mu \mathrm{l}$ PBS and subsequently subjected to DNA extraction as described above. Spiking of $A$. acidiphilus, $S$. ruber and $R$. radiobacter, and 454-pyrosequencing were performed according to the validation protocol described above. For these experiments, bacterial cells of $S$. ruber, $R$. radiobacter and $A$. acidiphilus equal to $3.0 \times 10^{8}, 5.0 \times 10^{8}$, $1.0 \times 10^{8} 16 \mathrm{~S}$ rDNA copies, respectively, were spiked into each crude sample.

\section{Amplification of V3-V6 16S rDNA variable region and 454 pyrosequencing}

Spike bacteria-specific qPCR was performed for all specimens (mice and human) to identify errors in DNA isolation before undergoing amplification and pyrosequencing (see Fig. 6).

A total of $25 \mathrm{ng}$ metagenomic DNA was used as a template to amplify the V3-V6 variable regions of the $16 \mathrm{~S}$ rRNA gene. PCR was performed using primer pair $341 \mathrm{~F}$ 1061R containing Lib-L adaptors and Roche standard mulitiplex identifiers (MIDs) in a final volume of $40 \mu \mathrm{l}$ containing $0.088 \mu \mathrm{M}$ of each primer, $2 \mathrm{mM} \mathrm{MgCl}_{2}$, and 1 U Platinum Taq DNA Polymerase (Life Technologies). The PCR amplification (see Fig. 6, step 4) was carried out over 30 cycles $\left(30 \mathrm{~s}\right.$ at $95{ }^{\circ} \mathrm{C}, 45 \mathrm{~s}$ at $64{ }^{\circ} \mathrm{C}, 45 \mathrm{~s}$ at $72{ }^{\circ} \mathrm{C}$ ) with an initial 5-min hot start at $95{ }^{\circ} \mathrm{C}$ and a final 


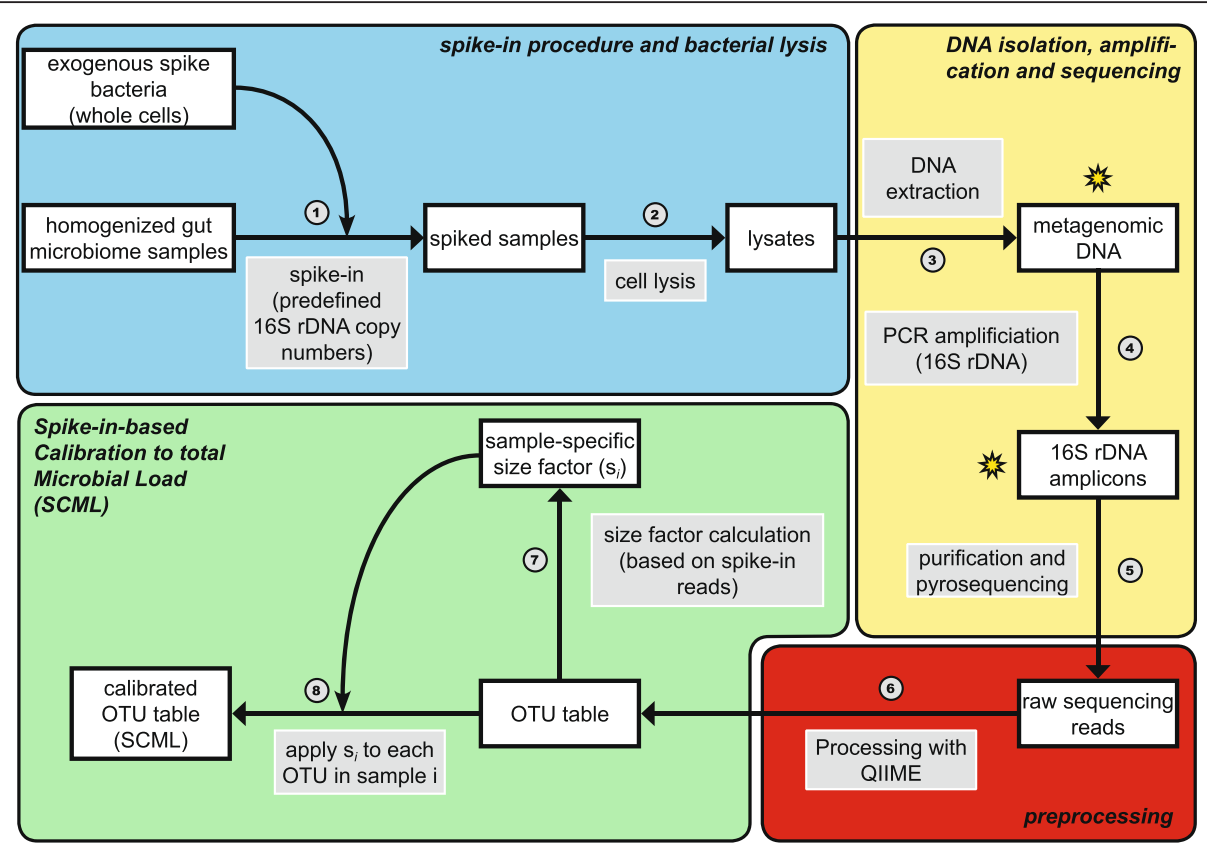

Fig. 6 Procedural overview of proposed spike-in procedure and the spike-in-based calibration to total microbial load (SCML). The overview is divided into four sections: spike-in procedure and bacterial lysis (blue), DNA isolation, amplification and sequencing (yellow), pre-processing (red) and the actual spikein-based calibration to microbial load (green). White-filled boxes depict procedural intermediates, while grey-filled boxes depict the different procedural steps. Each step is numbered. In the first step (1) whole cells of exogenous spike bacteria corresponding to a fixed number of 165 rDNA copies are added to homogenized microbiome samples. Bacterial lysis is performed on the resulting spiked samples (2). Metagenomic DNA is extracted from the lysates (3) and PCR amplified using 16S rDNA specific primers (4), creating $16 \mathrm{~S} \mathrm{rDNA}$ amplicons. These amplicons are purified and pyrosequencing is performed (5). The resulting raw read counts are pre-processed with QIIME (quality filtering, demultiplexing and closed reference OTU picking) to generate OTU read count tables (6). Based on the read counts associated with single or multiple reference spike-in bacteria, a size factor $\mathrm{s}_{\mathrm{i}}$ for each sample $\mathrm{i}$ is calculated and applied to each OTU of this particular sample i (8, see methods section). This leads to an OTU read count table calibrated to differences in microbial load. These read counts can be utilized to more accurately assess changes between different samples. All depicted steps are described in detail in the methods section. Stars indicate points in the procedure at which qPCR is performed to identify possible errors in DNA isolation (metagenomic DNA) or PCR amplification (16S rDNA amplicons).

extension step $\left(7 \mathrm{~min}\right.$ at $\left.72{ }^{\circ} \mathrm{C}\right)$. The resulting 790 -bp amplicons were analysed by standard agarose gel electrophoresis on a $1.5 \%(\mathrm{w} / \mathrm{v})$ gel. The amplicons were extracted from agarose gels using the QIAquick Gel Extraction Kit (Qiagen, Hilden, Germany) and purified with Agencourt AMPure XP beads (Beckman Coulter, Krefeld, Germany). Copy numbers of amplicons containing LibL-adaptors were determined using the KAPA Library Quant 454 Titanium/Lib-L Universal Kit (KAPA Biosystems, Wilmington, DE, USA) and pooled to a normalized library with a concentration of $1 \times 10^{7}$ adaptor-labeled amplicon molecules/ $\mu \mathrm{l}$ for each sample. This library was subjected to sequencing (see Fig. 6, step 5) using the GS FLX+ system (454/ Roche) and the GS FLX Titanium LV emPCR Kit (Lib-L) applying 0.4 copies per bead. Sequencing was performed on a full PTP according to manufacturer's protocol using the GS-FLX Titanium Sequencing Kit $\mathrm{XL}+$ and the acyclic flow pattern B. Sequencing raw data was processed with gsRunProcessor v2.9 (Roche) using quality filtering as defined by the default LongAmplicons 3 pipeline resulting in $895 \mathrm{Mb}$ from
1,313,653 passed filter wells with a median read length of 706 bases.

\section{Quantification of $16 \mathrm{~S}$ rRNA gene copy number by qRT-PCR Primer design and validation}

Primers and probes for quantification of eubacterial $16 \mathrm{~S}$ rDNA copies (Additional file 4: Table S4) were designed and evaluated in silico based on the RefNR sequence collection of the SILVA reference database release 119 [34] containing 534,968 16S rRNA sequences. The overall SILVA database coverage of universal 16S rDNA quantification primers $764 \mathrm{~F}$ and $907 \mathrm{R}$ allowing one primer mismatch was $86 \%$. Allowing no primer mismatches, specificity of primers and probes targeting $S$. ruber, $R$. radiobacter and A. acidiphilus DNA exhibited specificities of $100 \%$. Specificity of primers and probes were further evaluated in silico using the blastn algorithm against the nucleotide collection (nt) database. Concentration of primers were optimized by titration in the range of the kit manufacturer's recommendations after PCR amplification of $16 \mathrm{~S}$ rDNA targets from DNA extracts of human and murine faecal specimens. 
Samples were spiked prior to DNA extraction with defined cell counts of $S$. ruber, R. radiobacter and A. acidiphilus, which were quantified microscopically using a modified Neubauer counting chamber. PCR products were screened for nonspecific bands by agarose gel electrophoresis (probe based assays) or agarose gel and melting curve analysis (SYBR Green I based assays). Specificity was further evaluated by quantitative realtime PCR amplification of total $16 \mathrm{~S} \mathrm{rDNA}$ and $16 \mathrm{~S}$ rDNA of spike-in bacteria from ten non-spiked murine and human DNA extracts.

\section{Quantification of total 165 rDNA}

To verify the experimental design, $16 \mathrm{~S}$ rRNA gene copies of total and spike-in bacteria were determined by qRT-PCR on a LightCycler 480 II Instrument (Roche). Primers and probes used are shown in Additional file 4: Table S4. PCR reactions included $1 \mu \mathrm{M}$ each of eubacterial 16S rRNA gene primers 764 F and 907R (quantification primers) and the LightCycler 480 SYBR Green I Master Kit (Roche). Quantification standards were generated by cloning complex PCR amplicon mixtures that were generated from a caecal microbiome DNA preparation of wild type C57BL/6J mice (using primers $341 \mathrm{~F}$ and 1061R) into the pGEM-T.Easy vector (Promega, Madison, WI, USA). Cloning of PCR amplicon mixtures was carried out to mimic a complex murine microbiota with respect to qPCR amplification efficiency in analyzed samples as far as possible. Quantification PCR was conducted over 40 cycles $\left(95{ }^{\circ} \mathrm{C}\right.$ for $10 \mathrm{~s}, 60^{\circ} \mathrm{C}$ for $15 \mathrm{~s}$ and $72{ }^{\circ} \mathrm{C}$ for $15 \mathrm{~s}$ ) with an initial 10 -min hot start at $95{ }^{\circ} \mathrm{C}$.

\section{Quantification of 165 rDNA of spike-in bacteria}

$16 \mathrm{~S}$ rRNA gene copy numbers of the spike-in bacteria $S$. ruber, $R$. radiobacter and $A$. acidiphilus were determined with $16 \mathrm{~S}$ rDNA-targeted species-specific primers and hydrolysis probes (see Additional file 4: Table S4). Quantification PCR was conducted using the LightCycler 480 Probes Master kit (Roche) in a $20-\mu \mathrm{l}$ reaction volume containing $4 \mathrm{mM} \mathrm{MgCl}_{2}, 0.25 \mu \mathrm{M}$ of each primer, and $0.1 \mu \mathrm{M}$ probes. Quantification standards were constructed by cloning full length 16S PCR amplicons of all spike-in bacteria (amplified using $27 \mathrm{~F}$ and 1492R primers) into pGEM-T.Easy. Quantification PCR was conducted over 40 cycles $\left(95^{\circ} \mathrm{C}\right.$ for $30 \mathrm{~s}, 60{ }^{\circ} \mathrm{C}$ for $30 \mathrm{~s}$ and $72{ }^{\circ} \mathrm{C}$ for $30 \mathrm{~s}$ ) with an initial 10-min hot start at $95^{\circ} \mathrm{C}$.

\section{Computational analysis}

We used a combination of QIIME [11] (v1.8.0) and R version 3.2.0 [35] with installed Bioconductor package [36] to process the read data. Reads were filtered for quality using QIIME's split_libraries.py script (see Fig. 6, step 6) with default parameters except minimum and maximum read length, which were set to $400 \mathrm{bp}$ and
$800 \mathrm{bp}$, respectively. This read length threshold covered $99.99 \%$ of all sequencing reads. The filtered reads were mapped to OTUs built on the SILVA [34] database (release 111) using QIIME's pick_closed_reference_otus.py script (see Fig. 6, step 6) with default parameters. The reference database OTUs used here constituted computationally built clusters of the SILVA SSU (small subunit) ribosomal RNA database. The clustering (see Fig. 6, step 6) was achieved by UCLUST 1.2.20 [37] and provided by the QIIME team (available at http://qiime.org/home_static/dataFiles.html). Since reads from the three spikein bacteria mapped to multiple OTUs, due to multiple reference OTUs encoding for the same spike-in genus, we deleted all but one OTU encoding for each spike-in from the database before mapping, to accumulate all reads from the spike-in to just this one OTU. The used reference sequences for these three OTUs are available in Additional file 5. Raw sequencing data of the dilution experiment is deposited in the European Nucleotide Archive (ENA) under the study accession number PRJEB11953, at http://www.ebi.ac.uk/ena/data/view/ PRJEB11953. Details of the sample design are shown in Additional file 2: Table S2. Relative abundances were calculated by dividing each OTU read count by total read count of the corresponding sample.

Ratios of absolute abundances were calculated by using the expectation that the counts of reference spikeins are inversely correlated to total microbial load of the samples under investigation. Let $\bar{s}$ be the mean read count of the reference spike-in $S$. ruber over all samples (see Fig. 6, step 7). The read count of every OTU in a sample $_{i}$ is rescaled by a factor $s_{i}$ that is calibrated such that the spike-in count is equal to $\bar{s}$ in every sample (see Fig. 6, step 8). SCML can be performed by the use of an individual spike-in bacterium or the sum of all reads obtained for multiple spike-in bacteria. For further analysis, the counts are $\log _{2}$ transformed.

To compare ratios derived from relative abundances and those derived by SCML, we calculate $\log _{2}$ ratios between every pair of samples for each method as a symmetrical measure of difference. Ratios of relative abundances are calculated by dividing the relative abundances of each OTU by its relative abundance in the compared sample, whereas ratios for SCML are calculated by means of the spike-in calibrated read counts (SCML data). If for example OTU A shows relative abundances of $20 \%$ and $40 \%$ in samples 1 and 2, respectively, the corresponding ratio for this comparison would be $0.4 / 0.2=2$, i.e. the abundance of OTU A in sample 2 is two times higher than in sample 1 . The corresponding $\log _{2}$ ratio would be $\log _{2}(2)=1$. Both ratios are calculated separately for each OTU.

For the combination approach of SCML, the read counts of $A$. acidiphilus and $R$. radiobacter were 
adjusted by their difference in the predefined spike-in concentration (Additional file 2: Table S2) towards $S$. ruber. If for example $A$. acidiphilus was added by design in half the concentration compared to $S$. ruber, then all reads by $A$. acidiphilus were multiplied by two. The adjusted read counts of $A$. acidiphilus, $R$. radiobacter and the raw read counts of $S$. ruber were summed up to one artificial entity. These summed reads were used in the same fashion as the $S$. ruber read counts in the single spike-in calculation. For the dilution experiment the adjustment of $A$. acidiphilus and $R$. radiobacter read counts was necessary, because both spike-ins were added in varying amounts in this experiment. In an application of our spike-in procedure (e.g. ASCT specimens in this study) all spike-in bacteria cells are added at fixed amounts. Therefore, an adjustment of the spike-in read counts before the combination would be obsolete.

\section{Additional files}

\section{Additional file 1: Table S1: Species-specific and total 165 rDNA}

quantitative realtime-PCR measurements. (XLSX $13 \mathrm{~kb}$ )

Additional file 2: Table S2: Design of dilution experiment. (XLSX $16 \mathrm{~kb}$ )

Additional file 3: Table S3: Pools of bacterial mock communities containing S. ruber, A. acidiphilus and $R$. radiobacter. (XLSX $13 \mathrm{~kb}$ )

Additional file 4: Table S4: Primers and hydrolysis probes used in this study. (XLSX $13 \mathrm{~kb}$ )

Additional file 5: $16 \mathrm{~S}$ rDNA reference sequences of S. ruber, A. acidiphilus and $R$. radiobacter. (FASTA $4 \mathrm{~kb}$ )

Additional file 6: R-Script for reproduction of analysis and figures of this study. (R $39 \mathrm{~kb}$ )

Additional file 7: Dilution experiment mapping file as used for reproduction. (TXT $3 \mathrm{~kb}$ )

Additional file 8: $\mathrm{ASCT}$ experiment mapping file as used for reproduction. (TXT $1 \mathrm{~kb}$ )

Additional file 9: Dilution experiment OTU table as used for reproduction. (TXT $460 \mathrm{~kb}$ )

Additional file 10: ASCT experiment OTU table as used for reproduction. (TXT $621 \mathrm{~kb}$ )

Additional file 11: Total 165 rDNA gRT-PCR measurements as used for reproduction. (CSV $1 \mathrm{~kb})$

Additional file 12: Spike-in concentrations by design as used for reproduction. (CSV $1 \mathrm{~kb})$

\section{Abbreviations}

A. acidiphilus, Alicyclobacillus acidiphilus; ASCT, allogeneic stem cell transplantation; GI-GvHD, gastrointestinal graft-versus-host disease; OTU, operational taxonomic unit; qRT-PCR, quantitative real-time polymerase chain reaction; $R$. radiobacter, Rhizobium radiobacter; S. ruber, Salinibacter ruber; SCML, spike-in-based calibration to microbial load

\section{Acknowledgements}

The authors acknowledge the excellent assistance of Nadja Reul, Claudia Deinzer, Christine Irtenkauf and Holger Melzl with all experimental procedures.

\section{Funding}

$F S, R S, A G$, JG and AH received core funding support by the DFG priority program SPP 1656 from the German Research Foundation. EH and DW were partially supported by the Regensburg Centre for Interventional Immunology (RCI).

\section{Availability of data and materials}

Quantitative realtime-PCR measurements are shown in Additional file 1: Table S1. Additional file 2: Table S2 illustrates the design of the dilution experiment. A table with the six different spike-in pools is provided in Additional file 3: Table S3. Primers and hydrolysis probes used in this study, as well as their references, are listed in Additional file 4: Table S4. Additional file 5 contains the used 165 reference sequences of all three spike-in bacteria. A documented R-script and the data needed to reproduce figures and analyses are provided in Additional files 6, 7, 8, 9, 10, 11 and 12, respectively. Raw sequencing data of both dilution experiment and human ASCT specimens is deposited in the European Nucleotide Archive (ENA) under the study accession number PRJEB11953, at http://www.ebi.ac.uk/ena/data/view/PRJEB11953.

\section{Authors' contributions}

$F S, R S, A G, A H$ and JG designed the validation experiment and the spike-in protocol. Acquisition and processing of the mice samples for the validation experiment were performed by JG. AH performed qRT-PCR measurements and sequencing. EH and DW collected the human stool specimens and provided clinical information. FS performed the bioinformatics analysis and prepared the figures. FS, RS, PJO, JG and AH wrote the manuscript. All authors read and approved the final manuscript.

\section{Competing interests}

The authors declare that they have no competing interests.

\section{Consent for publication}

All patients consented in writing to the disclosure and publication of deidentified data.

\section{Ethics approval and consent to participate \\ Mice}

All mice were maintained under specific pathogen-free conditions at the central animal laboratories of the University Medical Centre of Regensburg. Experiments were carried out according to the EU Directive 2010/63/EU on the protection of animals used for scientific purposes as well as to the institutional guidelines for animal welfare.

Human

Sampling of human stool specimens, microbiome analysis and collection of clinical data has been approved by the Local Ethical Committee of the University Medical Centre of Regensburg (ethics application numbers: 02/220 and 09/059). All patients gave written informed consent prior to admission to ASCT.

\section{Author details}

${ }^{1}$ Chair of Statistical Bioinformatics, University of Regensburg, Am BioPark 9, 93053 Regensburg, Germany. ${ }^{2}$ Institute of Clinical Microbiology and Hygiene, University Medical Centre, Franz-Josef-Strauß-Allee 11, 93053 Regensburg, Germany. ${ }^{3}$ Department of Haematology and Oncology, Internal Medicine III, University Medical Centre, Franz-Josef-Strauß-Allee 11, 93053 Regensburg, Germany. ${ }^{4}$ Chair and Institute of Functional Genomics, University of Regensburg, Am BioPark 9, 93053 Regensburg, Germany.

Received: 14 December 2015 Accepted: 2 June 2016

Published online: 21 June 2016

\section{References}

1. Walker A, Pfitzner B, Neschen S, Kahle M, Harir M, Lucio M, et al. Distinct signatures of host-microbial meta-metabolome and gut microbiome in two C57BL/6 strains under high-fat diet. ISME J. 2014;8(12):2380-96. doi:10.1038/ ismej.2014.79.

2. Buffie CG, Pamer EG. Microbiota-mediated colonization resistance against intestinal pathogens. Nat Rev Immunol. 2013;13(11):790-801. doi:10.1038/nri3535.

3. Chung H, Pamp SJ, Hill JA, Surana NK, Edelman SM, Troy EB, et al. Gut immune maturation depends on colonization with a host-specific microbiota. Cell. 2012;149(7):1578-93. doi:10.1016/j.cell.2012.04.037.

4. Haberman Y, Tickle TL, Dexheimer PJ, Kim MO, Tang D, Karns R, et al. Pediatric Crohn disease patients exhibit specific ileal transcriptome and microbiome signature. J Clin Invest. 2014;124(8):3617-33. doi:10.1172/JCI75436. 
5. Kostic AD, Xavier RJ, Gevers D. The microbiome in inflammatory bowel disease: current status and the future ahead. Gastroenterology. 2014;146(6): 1489-99. doi:10.1053/j.gastro.2014.02.009.

6. lida N, Dzutsev A, Stewart CA, Smith L, Bouladoux N, Weingarten RA, et al. Commensal bacteria control cancer response to therapy by modulating the tumor microenvironment. Science. 2013;342(6161):967-70. doi:10.1126/ science.1240527.

7. Klimesova K, Kverka M, Zakostelska Z, Hudcovic T, Hrncir T, Stepankova R, et al. Altered gut microbiota promotes colitis-associated cancer in IL-1 receptor-associated kinase M-deficient mice. Inflamm Bowel Dis. 2013;19(6): 1266-77. doi:10.1097/MIB.0b013e318281330a.

8. Huse SM, Dethlefsen L, Huber JA, Welch DM, Relman DA, Sogin ML. Exploring Microbial Diversity and Taxonomy Using SSU rRNA Hypervariable Tag Sequencing. PLoS Genet. 2008:4(11):e1000255. doi:10. 1371/journal.pgen.1000255.

9. Claesson MJ, Wang Q, O'Sullivan O, Greene-Diniz R, Cole JR, Ross RP, et al. Comparison of two next-generation sequencing technologies for resolving highly complex microbiota composition using tandem variable 165 rRNA gene regions. Nucleic Acids Res. 2010;38(22):e200. doi:10.1093/nar/gkq873.

10. Ong SH, Kukkillaya VU, Wilm A, Lay C, Ho EX, Low L, et al. Species identification and profiling of complex microbial communities using shotgun Illumina sequencing of $16 \mathrm{~S}$ rRNA amplicon sequences. PLOS ONE. 2013;8(4):e60811. doi:10.1371/journal.pone.0060811.

11. Caporaso JG, Kuczynski J, Stombaugh J, Bittinger K, Bushman FD, Costello EK et al. QIIME allows analysis of high-throughput community sequencing data. Nat Methods. 2010;7(5):335-6. doi:10.1038/nmeth.f.303.

12. Schloss PD, Westcott SL, Ryabin T, Hall JR, Hartmann M, Hollister EB, et al. Introducing mothur: open-source, platform-independent, communitysupported software for describing and comparing microbial communities. Appl Environ Microbiol. 2009;75(23):7537-41. doi:10.1128/AEM.01541-09.

13. Blaxter M, Mann J, Chapman T, Thomas F, Whitton C, Floyd R, et al. Defining operational taxonomic units using DNA barcode data. Philos Trans R Soc Lond Ser B Biol Sci. 2005;360(1462):1935-43. doi:10.1098/rstb.2005.1725.

14. Turnbaugh PJ, Ridaura VK, Faith JJ, Rey FE, Knight R, Gordon J. The effect of diet on the human gut microbiome: a metagenomic analysis in humanized gnotobiotic mice. Sci Transl Med. 2009;1(6):6ra14. doi:10. 1126/scitranslmed.3000322.

15. Koren O, Knights D, Gonzalez A, Waldron L, Segata N, Knight R, et al. A guide to enterotypes across the human body: meta-analysis of microbial community structures in human microbiome datasets. PLoS Comput Biol. 2013;9(1):e1002863. doi:10.1371/journal.pcbi.1002863.

16. Holler E, Butzhammer P, Schmid K, Hundsrucker C, Koestler J, Peter K, et al, Metagenomic analysis of the stool microbiome in patients receiving allogeneic stem cell transplantation: loss of diversity is associated with use of systemic antibiotics and more pronounced in gastrointestinal graftversus-host disease. Biol Blood Marrow Transplant. 2014;20(5):640-5. doi:10. 1016/j.bbmt.2014.01.030.

17. Risso D, Ngai J, Speed TP, Dudoit S. Normalization of RNA-seq data using factor analysis of control genes or samples. Nat Biotechnol. 2014;32(9):896902. doi:10.1038/nbt.2931.

18. Geiger T, Wisniewski JR, Cox J, Zanivan S, Kruger M, Ishihama $Y$, et al. Use of stable isotope labeling by amino acids in cell culture as a spikein standard in quantitative proteomics. Nat Protoc. 2011;6(2):147-57. doi:10.1038/nprot.2010.192.

19. Wu L, Mashego MR, van Dam JC, Proell AM, Vinke JL, Ras C, et al. Quantitative analysis of the microbial metabolome by isotope dilution mass spectrometry using uniformly 13C-labeled cell extracts as internal standards. Anal Biochem. 2005;336(2):164-71. doi:10.1016/j.ab.2004.09.001.

20. Jones MB, Highlander SK, Anderson EL, Li W, Dayrit M, Klitgord N, et al. Library preparation methodology can influence genomic and functional predictions in human microbiome research. Proc Natl Acad Sci U S A. 2015; 112(45):14024-9. doi:10.1073/pnas.1519288112.

21. Anton J, Oren A, Benlloch S, Rodriguez-Valera F, Amann R, Rossello-Mora R. Salinibacter ruber gen. nov., sp. nov., a novel, extremely halophilic member of the Bacteria from saltern crystallizer ponds. Int J Syst Evol Microbiol. 2002; 52(Pt 2):485-91. doi:10.1099/00207713-52-2-485.

22. Zhang L, Li X, Zhang F, Wang G. Genomic analysis of Agrobacterium radiobacter DSM 30147(T) and emended description of A. radiobacter (Beijerinck and van Delden 1902) Conn 1942 (Approved Lists 1980) emend. Sawada et al. 1993. Stand Genomic Sci. 2014;9(3):574-84. doi:10. 4056/sigs.4688352.
23. Matsubara H, Goto K, Matsumura T, Mochida K, Iwaki M, Niwa M, et al. Alicyclobacillus acidiphilus sp nov., a novel thermo-acidophilic, omegaalicyclic fatty acid-containing bacterium isolated from acidic beverages. Int J Syst Evol Microbiol. 2002;52(Pt 5):1681-5. doi:10.1099/ijs.0.02169-0.

24. Jenq RR, Ubeda C, Taur Y, Menezes CC, Khanin R, Dudakov JA, et al. Regulation of intestinal inflammation by microbiota following allogeneic bone marrow transplantation. J Exp Med. 2012;209(5):903-11. doi:10.1084/ jem.20112408.

25. Weber D, Oefner PJ, Hiergeist A, Koestler J, Gessner A, Weber M, et al. Low urinary indoxyl sulfate levels early after transplantation reflect a disrupted microbiome and are associated with poor outcome. Blood. 2015;126(14): 1723-8. doi:10.1182/blood-2015-04-638858.

26. Brukner I, Longtin $Y$, Oughton $M$, Forgetta $V$, Dascal A. Assay for estimating total bacterial load: relative $\mathrm{QPCR}$ normalisation of bacterial load with associated clinical implications. Diagn Microbiol Infect Dis. 2015;83(1):1-6. doi:10.1016/j.diagmicrobio.2015.04.005.

27. Amend AS, Seifert KA, Bruns TD. Quantifying microbial communities with 454 pyrosequencing: does read abundance count? Mol Ecol. 2010;19(24): 5555-65. doi:10.1111/j.1365-294X.2010.04898.x.

28. Costello EK, Stagaman K, Dethlefsen L, Bohannan BJ, Relman DA. The application of ecological theory toward an understanding of the human microbiome. Science. 2012;336(6086):1255-62. doi:10.1126/science.1224203.

29. Cho I, Blaser MJ. The human microbiome: at the interface of health and disease. Nat Rev Genet. 2012;13(4):260-70. doi:10.1038/nrg3182.

30. Walter J, Ley R. The human gut microbiome: ecology and recent evolutionary changes. Annu Rev Microbiol. 2011;65:411-29. doi:10.1146/ annurev-micro-090110-102830.

31. Aitchison J. A New Approach to Null Correlations of Proportions. J Int Ass Math Geol. 1981;13(2):175-89. doi:10.1007/Bf01031393.

32. Aitchison J. The Statistical-Analysis of Compositional Data. J Roy Stat Soc B Met. 1982;44(2):139-77.

33. Stoddard SF, Smith BJ, Hein R, Roller BR, Schmidt TM. rrnDB: improved tools for interpreting rRNA gene abundance in bacteria and archaea and a new foundation for future development. Nucleic Acids Res. 2015;43(Database issue):D593-8. doi:10.1093/nar/gku1201.

34. Quast C, Pruesse E, Yilmaz P, Gerken J, Schweer T, Yarza P, et al. The SILVA ribosomal RNA gene database project: improved data processing and web-based tools. Nucleic Acids Res. 2013;41(Database issue):D5906. doi:10.1093/nar/gks1219.

35. RCoreTeam. R: A Language and Environment for Statistical Computing Vienna: R Foundation for Statistical Computing; 2015. http://www.rproject.org. Accessed 10 Dec 2015.

36. Huber W, Carey VJ, Gentleman R, Anders S, Carlson M, Carvalho BS, et al. Orchestrating high-throughput genomic analysis with Bioconductor. Nat Methods. 2015;12(2):115-21. doi:10.1038/nmeth.3252.

37. Edgar RC. Search and clustering orders of magnitude faster than BLAST. Bioinformatics. 2010;26(19):2460-1. doi:10.1093/bioinformatics/btq461.

\section{Submit your next manuscript to BioMed Central and we will help you at every step:}

- We accept pre-submission inquiries

- Our selector tool helps you to find the most relevant journal

- We provide round the clock customer support

- Convenient online submission

- Thorough peer review

- Inclusion in PubMed and all major indexing services

- Maximum visibility for your research

Submit your manuscript at www.biomedcentral.com/submit
(O) BioMed Central 